\title{
Tracking nuclear wave-packet dynamics in molecular oxygen ions with few-cycle infrared laser pulses
}

\author{
S. De, ${ }^{1,{ }^{*}}$ I. A. Bocharova, ${ }^{1}$ M. Magrakvelidze,${ }^{1}$ D. Ray, ${ }^{1}$ W. Cao, ${ }^{1}$ B. Bergues,${ }^{2}$ U. Thumm, ${ }^{1}$ M. F. Kling, ${ }^{1,2}$ \\ I. V. Litvinyuk, ${ }^{1,3}$ and C. L. Cocke ${ }^{1, \dagger}$ \\ ${ }^{1}$ J. R. Macdonald Laboratory, Physics Department, Kansas State University, Manhattan, Kansas 66506, USA \\ ${ }^{2}$ Max Planck Institute of Quantum Optics, Hans-Kopfermann-Strasse 1, D-85748 Garching, Germany \\ ${ }^{3}$ Centre for Quantum Dynamics, Griffith University, Nathan, Queensland 4111, Australia
}

(Received 29 March 2010; published 14 July 2010)

\begin{abstract}
We have tracked nuclear wave-packet dynamics in doubly charged states of molecular oxygen using few-cycle infrared laser pulses. Bound and dissociating wave packets were launched and subsequently probed via a pair of 8 -fs pulses of $790 \mathrm{~nm}$ radiation. Ionic fragments from the dissociating molecules were monitored by velocity-map imaging. Pronounced oscillations in the delay-dependent kinetic energy release spectra were observed. The occurrence of vibrational revivals permits us to identify the potential curves of the $\mathrm{O}_{2}$ dication which are most relevant to the molecular dynamics. These studies show the accessibility to the dynamics of such higher-charged molecules.
\end{abstract}

DOI: 10.1103/PhysRevA.82.013408

PACS number(s): $34.50 . \mathrm{Rk}, 31.70 . \mathrm{Hq}, 33.80 . \mathrm{Rv}$

\section{INTRODUCTION}

The study of wave-packet dynamics of diatomic molecules in real time, carried out using a pump-probe approach, was pioneered two decades ago by Zewail et al. [1]. Early studies focused on weakly bound and/or heavy molecules for which the vibrational periods were longer than the several-hundredfemtosecond laser pulses available at that time. The rapid advance of laser technology has allowed the generation of infrared (IR) pulses down to 4 fs [2], which makes the probing of vibrational motion in even the fastest diatomic molecules possible. For example, vibrational dynamics in $\mathrm{H}_{2}$ and $\mathrm{D}_{2}$ (with vibrational periods around 20 and $30 \mathrm{fs,}$ respectively) have been studied by producing the molecular cations of these species with short (8-10 fs) pulses of $800 \mathrm{~nm}$ wavelength [3-5]. The nuclear wave-packet motion was probed in these experiments by ionization of the molecular cations via a second, delayed laser pulse. The observed vibrational motion took place predominantly on the two lowest potential curves of the molecular cations [3-5]. Here, the lower, bound state gives rise to an oscillating wave packet and the higher, repulsive state produces a dissociating wave packet. The extension of this approach to iodine has been reported [6], but for most molecules this extension is not straightforward because of the large number of molecular potential curves involved. In this paper, we report on few-cycle IR pump-IR probe measurements of nuclear wave-packet motion in various charge states of molecular oxygen. By selection of the final fragmentation channels we are able to track the wave-packet dynamics in the dication of $\mathrm{O}_{2}$ and to identify, through observation of the oscillation times and vibrational revival times, the particular states involved.

\section{EXPERIMENTAL RESULTS}

A strong IR pump pulse $(790 \mathrm{~nm}, 8 \mathrm{fs})$ of the order of $4 \times 10^{14} \mathrm{~W} / \mathrm{cm}^{2}$ is used to ionize and excite $\mathrm{O}_{2}$ molecules

\footnotetext{
*sankar@phys.ksu.edu

†cocke@phys.ksu.edu
}

ejected from an effusive source. After a delay of up to $1700 \mathrm{fs}$, an IR probe pulse with the same properties is used to ionize the system further and the fragments are detected using a velocity-map imaging spectrometer. The $3 \mathrm{D}$ momentum distributions of different charge states of fragment ions are obtained from the raw data by inverse Abel transformation via an iterative procedure [7]. Although no coincident detection of the partner fragment is made, the charge state of the partner ion can be deduced from the measured kinetic energy release (KER) spectra.

We first establish that the pump creates a substantial population of identifiable states in the dication of $\mathrm{O}_{2}$. Figure 1(a) shows a typical momentum spectrum of $\mathrm{O}^{+}$ions for a delay of $500 \mathrm{fs}$ between the laser pulses. This spectrum was observed not to depend strongly on pump-probe delay and thus shows the "primary" population in the parent molecular ion. The momentum spectrum is dominated by two "rings" with radii near 50 and 100 a.u. The corresponding KER spectrum obtained via angular integration of the momentum spectrum within a range of $30^{\circ}$ in the direction perpendicular to the laser polarization is shown in Fig. 1(b). We identify the contributions in the spectra as follows: The innermost ring and events at lower momenta in Fig. 1(a), corresponding to a KER of less than $2 \mathrm{eV}$, result from the dissociation of $\mathrm{O}_{2}^{+}$into $\mathrm{O}^{+}$and $\mathrm{O}$ fragments. Some of the low-energy features seen here were previously reported by Sayler et al. [8]. The ring with momenta near 100 a.u. in Fig. 1(a) results from the fragmentation of $\mathrm{O}_{2}{ }^{2+}$ into $\mathrm{O}^{+}$and $\mathrm{O}^{+}$fragments with KER between 6 and $13 \mathrm{eV}$. The higher KER originates mainly from the Coulomb repulsion between the two ions. The distinct lines in the spectrum of $\mathrm{O}^{+}$fragments correspond to well-defined initial states of the dication and have been observed and identified previously in both electron-induced [9] and laser-induced ionization [10]. The assignment of the states corresponding to the high-energy peaks in Fig. 1(b) is based on the work of Lundqvist et al. [9].

Figure 2(a) shows potential energy curves for $\mathrm{O}_{2}, \mathrm{O}_{2}{ }^{+}$, and $\mathrm{O}_{2}{ }^{2+}$ adapted from Refs. [9,11]. An expanded view of the region of the excited states of $\mathrm{O}_{2}{ }^{2+}$ is shown in Fig. 2(b). A Franck-Condon transition from the ground state of $\mathrm{O}_{2}$ will populate both quasibound and dissociative states in the dication. 

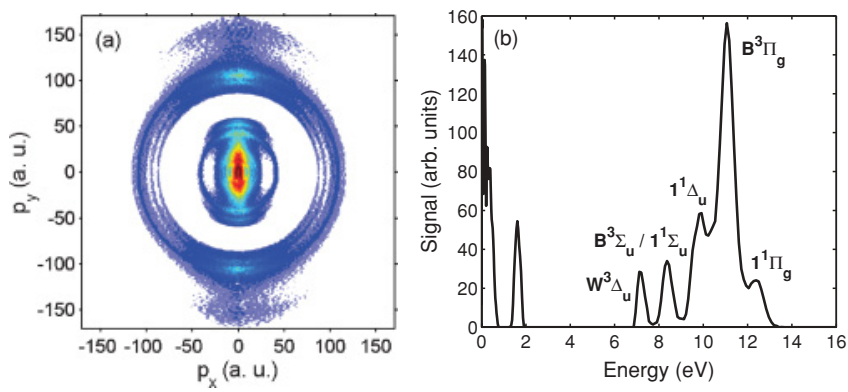

FIG. 1. (Color online) (a) Two-dimensional cut (along $p_{z}=0$ ) through the 3D momentum distribution in Cartesian coordinates $p_{x}$, $p_{y}$, and $p_{z}$ of $\mathrm{O}^{+}$ions from $\mathrm{O}_{2}$ at a delay of $500 \mathrm{fs}$. The polarization is parallel to the $p_{y}$ direction. (b) KER spectrum obtained from (a) via angular integration within a $30^{\circ}$ range in the direction perpendicular to the laser polarization.

The dissociation takes place on a picosecond to microsecond time scale [9] through tunneling or predissociation.

The nuclear wave-packet dynamics in $\mathrm{O}_{2}{ }^{2+}$ is studied by measuring the yield of $\mathrm{O}^{2+}$ fragment ions. Figure 3(a) shows a plot of the KER of $\mathrm{O}^{2+}$ ions as a function of the pump-probe delay. The plotted KER is obtained by angular integration of the momentum spectrum within a range of $90^{\circ}$ along the direction of the laser polarization. The two main features in Fig. 3 are qualitatively similar to what has been reported for the simple model systems $\mathrm{H}_{2}{ }^{+}$and $\mathrm{D}_{2}{ }^{+}[3,4]$ : (1) dissociative states that are populated by the pump pulse result in a monotonic decrease in KER with delay, and (2) the population of states that are bound (on a few-picosecond time scale) leads to bands of nearly constant KER. The modulations visible in these bands reflect the bound wave-packet motion as detailed below.

The strong band between 15 and $25 \mathrm{eV}$ can be attributed mainly to quasibound states of $\mathrm{O}_{2}{ }^{2+}$ produced by the pump and further ionized by the probe pulse to $\mathrm{O}_{2}{ }^{3+} \rightarrow \mathrm{O}^{2+}+\mathrm{O}^{+}$. The repetitive structure with a period near 33 fs along this band results from the wave-packet motion in the potential wells shown in Fig. 2(b). The expected vibrational periods $T_{\text {osc }}^{[9]}$ for the electronic states most relevant to the observed dynamics are given in Table I. Vibrational periods in the range

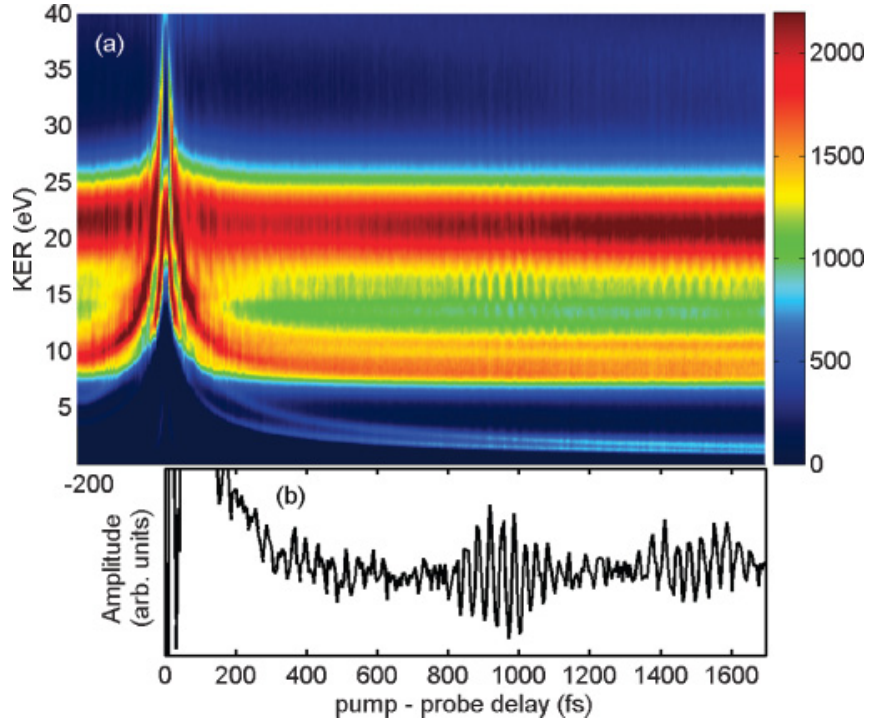

FIG. 3. (Color online) (a) KER (eV) vs pump-probe delay (fs) for $\mathrm{O}^{2+}$ ions. (b) Oscillations in the yield of $\mathrm{O}^{2+}$ for KERs between 15 and $17 \mathrm{eV}$ showing vibrational revival times near 955 and $1490 \mathrm{fs}$.

30-35 fs are characteristic of nearly all of these states. Despite the variation in the vibrational periods of the individual $\mathrm{O}_{2}{ }^{2+}$ states, stripes at early delay times up to ca. $300 \mathrm{fs}$ in the band between 15 and $25 \mathrm{eV}$ are visible. Similar to the previous experiments on molecular hydrogen $[3,4]$, stripes, rather than sinusoidal oscillations, are seen because the outgoing wave packet remains relatively localized on the outward trip to the turning point, becoming diffuse on the return trip after reflection and regrouping again upon reflection at the inner turning point. In the experimental data on $\mathrm{O}_{2}$ shown in Fig. 3(a), the stripes remain reasonably well pronounced for quite a number of trips before gradually becoming washed out due to the anharmonicity of the potential wells in which the motion occurs.

\section{ANALYSIS AND DISCUSSION}

In order to further analyze the nuclear dynamics as in the work of Thumm et al. [12,13], the power spectrum of Fig. 3 is

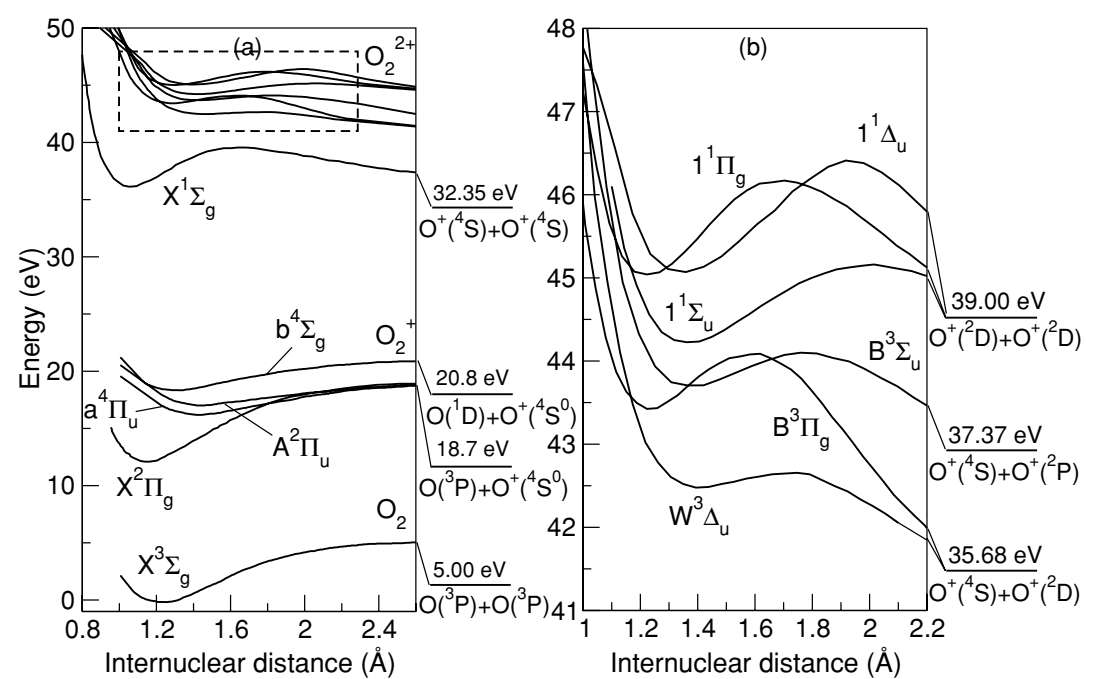

013408-2
FIG. 2. (a) Potential energy curves for $\mathrm{O}_{2}$ and $\mathrm{O}_{2}{ }^{+}$[11] and $\mathrm{O}_{2}{ }^{2+}$ [9]. (b) Expanded view of the region denoted in (a) by the dashed box. 
shown in Fig. 4(a). As pointed out in Ref. [13], such a power spectrum can in principle be used to deduce information about the shape of the potential curves involved in the dynamics. The strong group of peaks appearing at a frequency close to $30 \mathrm{THz}$ in Fig. 4 corresponds to the motion in the $1^{1} \Delta_{u}$, $1^{1} \Sigma_{u}, \mathrm{~B}{ }^{3} \Sigma_{u}$, and $\mathrm{W}^{3} \Delta_{u}$ potential wells. The main single reason these wells are expected to dominate in the Fourier spectrum over other states of the dication is that they have equilibrium distances far from that of the neutral molecule and thus are created in high vibrational states, giving rise to distinct oscillating wave packets. Since these wells are not harmonic, the vibrational frequency goes down with increasing vibrational quantum number. One expects to see this revealed in the power spectrum as well-resolved distinct vibrational frequency differences. Such a structure is seen in the group near $30 \mathrm{THz}$, although the experimental resolution is limited by the maximum delay range of $1.7 \mathrm{ps}$. Another weak structure appears close to $56 \mathrm{THz}$, which we interpret as due to the motion in the $\mathrm{X}^{1} \Sigma_{g}$ ground-state potential of the dication. This state is created mainly in its vibrational ground state, however, and the wave-packet oscillation is weak. In contrast to the earlier work on molecular hydrogen, the derivation of the exact shape of the potential wells of the states involved in the nuclear dynamics is not possible both due to the large number of states involved and due to the fact that "Coulomb explosion" of $\mathrm{O}_{2}{ }^{3+}$ occurs along multiple unidentified potential curves.

Identification of the individual states of $\mathrm{O}_{2}{ }^{2+}$ contributing to the observed dynamics becomes possible via their characteristic vibrational revival times. Figure 3 (b) shows the oscillations of the $\mathrm{O}^{2+}$ ion yield as a function of pump-probe delay. The curve was obtained by integration over an energy band between 15 and $17 \mathrm{eV}$. Two revivals are clearly seen at delays of $955 \pm 15$ and $1490 \pm 20$ fs, respectively. In Table I, we list the expected vibrational revival times for the four active states of $\mathrm{O}_{2}{ }^{2+}$ identified in Fig. 1(b). We obtain these in two ways. First, Lundquist et al. [9] listed values of $\omega x$ ( $\omega$ is the vibrational frequency and $x$ the anharmonicity parameter) for these states, from which the revival time $T_{\text {rev }}^{[9]}$ is $2 \pi /(\omega x)$. Second, we have performed Morse potential fits to the theoretical curves published in Ref. [9], from which the revival times $T_{\mathrm{rev}}^{\mathrm{Mor}}$
TABLE I. The vibrational frequency $v_{o}^{[9]}(\mathrm{THz})$, period $T_{\mathrm{osc}}^{[9]}$, and the revival time $T_{\text {rev }}^{[9]}$ for four relevant metastable states of $\mathrm{O}_{2}{ }^{2+}$.

\begin{tabular}{lcccc}
\hline \hline State & ${ }^{1} \Delta_{u}$ & ${ }^{1} \Sigma_{u}$ & $\mathrm{~B}^{3} \Sigma_{u}$ & $\mathrm{~W}^{3} \Delta_{u}$ \\
\hline$\nu_{o}^{[9]}(\mathrm{THz})$ & $32.1,35.3$ & $30.5,29.6$ & $28.2,31.7$ & $18.6,25.1$ \\
$T_{\text {osc }}^{[9]}(\mathrm{fs})^{\mathrm{a}}$ & $31.1,28.2$ & $32.7,33.6$ & $35.4,31.4$ & $53.7,39.7$ \\
$T_{\text {rev }}^{[9]}(\mathrm{fs})$ & 2381,980 & 1333,1515 & 926,833 & $\ldots, 595$ \\
$T_{\text {rev }}^{\text {Mor }}(\mathrm{fs})$ & 1536 & 983 & 864 & \\
$T_{\text {rev }}^{\text {exp }}(\mathrm{fs})$ & $1490(20)$ & $955(15)$ & $955(15)$ & {$[480]$} \\
\hline \hline
\end{tabular}

$\bar{a}$ The first and second values in the corresponding rows are derived from theory and spectroscopic data, respectively (adapted from Ref. [9]). $T_{\mathrm{rev}}^{\mathrm{Mor}}$ is the vibrational revival time analytically deduced by fitting Morse potentials to the published theoretical curves [9]. $T_{\mathrm{rev}}^{\mathrm{expt}}$ are vibrational revival times measured in the present experiment. The value in square brackets is tentative assignment.

can be deduced analytically. The values obtained from these fits differ somewhat from those given in Ref. [9] but are probably not inconsistent within the uncertainties of the fitting procedure. The experimental values of Ref. [9] do not bear error bars, but the error bars would probably be larger than the disagreement between the theory and the experiment. We therefore prefer to compare our results with the revival times extracted from the Morse fit. Based on this comparison, we identify the revival time measured at $955 \mathrm{fs}$ as due to the $1^{1} \Sigma_{u}$ (or B ${ }^{3} \Sigma_{u}$ ) state and that at 1490 fs due to the $1^{1} \Delta_{u}$ state. The corresponding theoretical revival times are 983 (or 864) and $1536 \mathrm{fs}$, respectively. Both $1^{1} \Sigma_{u}$ and B ${ }^{3} \Sigma_{u}$ states can contribute to the observed revival at $955 \mathrm{fs}$, although the prediction for the $1^{1} \Sigma_{u}$ state matches the theoretical result better. For the $\mathrm{W}^{3} \Delta_{u}$ state, the revival time deduced from the experiment of Ref. [9] is 595 fs. Although it is not nearly as clear as the other two, we do observe a weak revival near $480 \mathrm{fs}$ in our data [shown in Fig. 3(b)]. The absence of evidence for the $\mathrm{B}^{3} \Pi_{g}$ and $1{ }^{1} \Pi_{g}$ states (expected oscillation and revival times near 37 and 683 fs, respectively) in Figs. 3 and 4 may be due to the fact that these states are populated mainly in their vibrational ground states, as discussed in Ref. [9]. We note that the revival times deduced from the spectroscopic
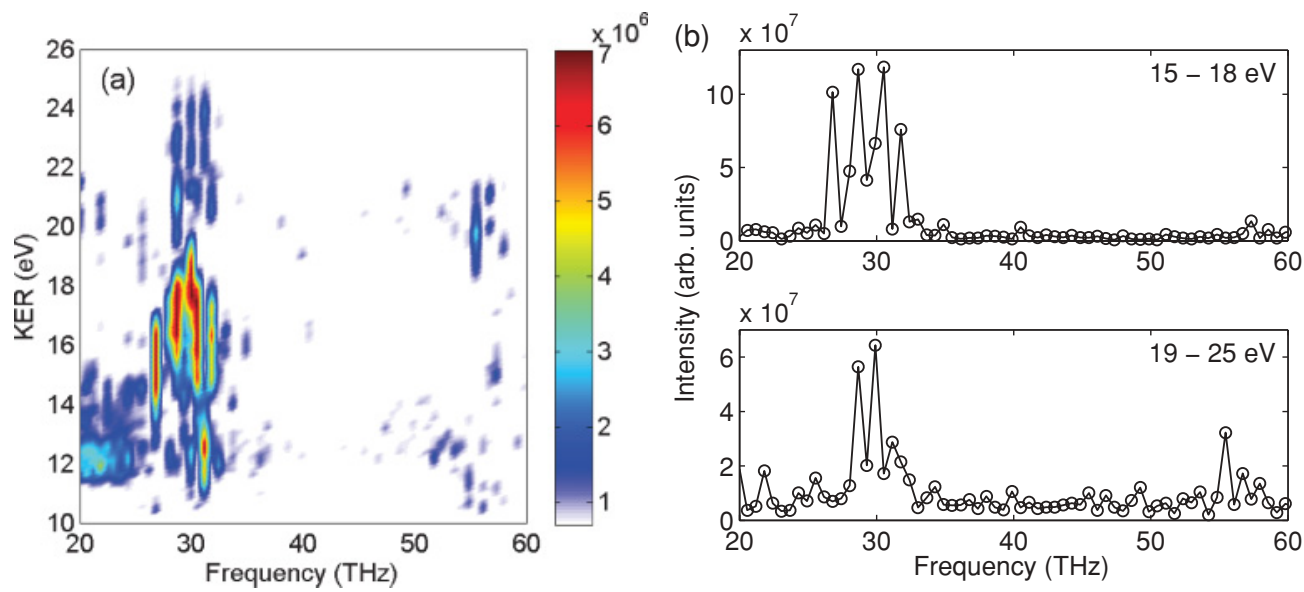

FIG. 4. (Color online) (a) The Fourier transform of the spectrum of Fig. 3(a): KER vs quantum-beat frequency. (b) Slices through energy bands in (a). 
data of Lundquist et al. (second value of $T_{\text {rev }}^{[9]}$ in Table I) are based on the dependence of the observed vibrational frequency spacing on the vibrational quantum number. Since only a few of such spacings (typically $<4$ ) were observed in these experiments [9], the revival times cannot be determined very precisely. In contrast, the clarity of the revivals in the present data allows an accurate extraction of the revival times. Indeed, our ability to identify the source of the very clear revivals strongly supports our identification of the states involved in the dynamics we observe.

It is important to note that we have assumed that the dynamics observed in the band between 15 and $25 \mathrm{eV}$ in Fig. 3(a) is due to the dication states. We cannot completely rule out the contributions from the cation and the neutral $\mathrm{O}_{2}$ molecule since they can also be ionized by the probe to produce $\mathrm{O}_{2}{ }^{3+}$. The calculated frequencies and revival times for the electronic ground state and the relevant exited states of $\mathrm{O}_{2}$ and $\mathrm{O}_{2}{ }^{+}$, however, do not match the experimental revival times observed here as well as those for the dication states. Therefore, we conclude that if there are such contributions, they at least do not dominate. A coincidence experiment as described in Ref. [14] would help us in identifying the contributions from each molecular ion separately.

\section{SUMMARY}

In summary, we have studied nuclear wave-packet dynamics in $\mathrm{O}_{2}$ molecular ions. The observation of vibrational revivals enabled us to identify the major states contributing to the bound-state wave-packet motion in the dication of $\mathrm{O}_{2}$. For two of the potential energy curves, we could extract precise vibrational revival times from the measured data. In view of the complexity of the molecular system, it is remarkable that such precise information can be extracted from time-resolved studies. Our work opens up a promising route towards detailed investigations of the dynamics of highly charged (complex) molecular species, which is also highly relevant to the experiments using free-electron lasers.

\section{ACKNOWLEDGMENTS}

We acknowledge B. Gaire and I. Ben-Itzhak for helpful discussions. The work was supported by the Chemical Sciences, Geosciences, and Biosciences Division, Office of Basic Energy Sciences, Office of Science, US Department of Energy, the National Science Foundation under Grant No. CHE-0822646 and the DFG via the Emmy-Noether program and the Cluster of Excellence: Munich Center for Advanced Photonics.
[1] A. H. Zewail, Science 242, 1645 (1988).

[2] A. L. Cavalieri et al., New J. Phys. 9, 242 (2007).

[3] T. Ergler, A. Rudenko, B. Feuerstein, K. Zrost, C. D. Schroter, R. Moshammer, and J. Ullrich, Phys. Rev. Lett. 97, 193001 (2006).

[4] A. S. Alnaser et al., Phys. Rev. A 72, 030702(R) (2005).

[5] H. Niikura, D. M. Villeneuve, and P. B. Corkum, Phys. Rev. A 73, 021402(R) (2006).

[6] L. Fang and G. N. Gibson, Phys. Rev. A 75, 063410 (2007).

[7] M. Vrakking, Rev. Sci. Instrum. 72, 4084 (2001).
[8] A. M. Sayler, P. Q. Wang, K. D. Carnes, B. D. Esry, and I. Ben-Itzhak, Phys. Rev. A 75, 063420 (2007).

[9] M. Lundqvist et al., J. Phys. B 29, 499 (1996).

[10] S. Voss et al., J. Phys. B 37, 4239 (2004).

[11] F. R. Gilmore, J. Quant. Spectrosc. Radiat. Transfer 5, 369 (1965).

[12] U. Thumm, T. Niederhausen, and B. Feuerstein, Phys. Rev. A 77, 063401 (2008).

[13] B. Feuerstein, T. Ergler, A. Rudenko, K. Zrost, C. D. Schroter, R. Moshammer, J. Ullrich, T. Niederhausen, and U. Thumm, Phys. Rev. Lett. 99, 153002 (2007).

[14] I. A. Bocharova, Ph.D. thesis, Kansas State University, 2009. 